\title{
Péritonite biliaire sur une cholécystite emphysémateuse et abcès hépatique à Clostridium perfringens
}

\author{
Biliary Peritonotis with an Emphysematous Cholecystitis and Liver Abcess Due \\ to Clostridium perfringens
}

\author{
C. Simon · J. Oliveira $\cdot$ X. Fontaine \\ Reçu le 24 avril 2018; accepté le 19 août 2018 \\ (C) SFMU et Lavoisier SAS 2018
}

\section{Introduction}

Les péritonites biliaires sont liées à l'évolution défavorable de cholécystites et d'angiocholites. Les cholécystites emphysémateuses sont des pathologies graves et rares, dont la prévalence est estimée à $3 \%$ [1]. Leur aspect scanographique " aérique » est caractéristique et souvent imputable aux bactéries anaérobies et aux bacilles à Gram négatif du tube digestif. Lorsqu'il existe une bactériémie associée, le taux de mortalité de cette pathologie peut atteindre 70 à $100 \%$ en l'absence de prise en charge médicochirurgicale adaptée [2]. Dans les cas les plus évolués, ces cholécystites emphysémateuses peuvent se compliquer d'abcès hépatiques par contiguïté ou translocation bactérienne intraabdominale [3]. Clostridum perfringens dont il est question ici est un bacille à Gram positif anaérobie strict présent dans le tube digestif chez des porteurs sains mais à risque pathogène d'autant plus s'il sécrète des exotoxines.

\section{Cas clinique}

Un homme de 59 ans est adressé aux urgences pour déséquilibre d'un diabète de découverte récente avec altération de l'état général et myalgies diffuses. À l'admission, le patient est apyrétique, asthénique, avec un teint gris et marbré. La fréquence cardiaque est à $120 / \mathrm{min}$ et la tension artérielle à 160/83 mmHg. L'auscultation cardiaque est normale. L'ECG retrouve une tachycardie sinusale et des ondes $T$ plates de façon diffuse. La glycémie capillaire est à 4,63 g/l, et la cétonémie capillaire est positive à $5,8 \mathrm{mmol} / \mathrm{l}$. Le patient présente une polypnée superficielle avec une saturation pul-

C. Simon $(\varangle) \cdot$ J. Oliveira $\cdot$ X. Fontaine

Service d'accueil des urgences, Smur,

hôpital Manchester, 45, avenue de Manchester,

F-08000 Charleville-Mézières, France

e-mail : cam08.simon@hotmail.fr sée en oxygène à $98 \%$ en air ambiant et une auscultation pulmonaire normale. Il n'existe pas de transit depuis une semaine, et l'examen abdominal est sans particularité.

Biologiquement, on note une acidose métabolique à trou anionique augmenté compatible avec une acidocétose diabétique $\left(\mathrm{pH}\right.$ à 7,21, $\mathrm{PaO}_{2}$ à $102 \mathrm{mmHg}, \mathrm{PaCO}_{2}$ à $16 \mathrm{mmHg}$, bicarbonates à $6,9 \mathrm{mmol} / \mathrm{l})$. La lactatémie est normale. L'ionogramme sanguin retrouve une hypokaliémie modérée à $3,06 \mathrm{mmol} / 1$ et une insuffisance rénale aiguë à diurèse conservée. Le bilan hépatique révèle une cytolyse (ALAT à $130 \mathrm{UI} / 1$, ASAT à $296 \mathrm{UI} / 1)$ et une cholestase ictérique (gamma-GT à $62 \mathrm{UI} / 1$, phosphatases alcalines à $122 \mathrm{UI} / 1$ et bilirubine totale à $73,3 \mathrm{mg} / \mathrm{l})$.

Une réhydratation intraveineuse est débutée avec recharge potassique par $\mathrm{KCl}$ à la seringue électrique. Une antibiothérapie probabiliste par ceftriaxone et métronidazole est initiée après réalisation des prélèvements microbiologiques au vu d'un syndrome inflammatoire biologique majeur (CRP à $455 \mathrm{mg} / \mathrm{l}$, procalcitonine à $12 \mathrm{ng} / \mathrm{ml}$, hyperleucocytose à 35 G/1). L'évolution clinique est marquée par une hyperthermie et un état de choc septique. Un scanner thoracoabdominopelvien met en évidence une cholécystite emphysémateuse avec aérobilie associée à un abcès hépatique périvésiculaire (segment IV) ainsi qu'un épanchement intra-abdominal et un pneumopéritoine (Fig. 1). Une prise en charge chirurgicale immédiate est décidée ainsi qu'une modification de l'antibiothérapie par pipéracilline-tazobactam et amikacine. L'intervention chirurgicale par laparotomie retrouve une vésicule gangréneuse perforée justifiant une cholécystectomie, une mise à plat de l'abcès hépatique et un lavage péritonéal. Des prélèvements bactériologiques peropératoires du liquide péritonéal et de l'abcès hépatique ainsi qu'une nouvelle série d'hémocultures sont réalisés. En réanimation, l'évolution est marquée par une instabilité hémodynamique réfractaire au remplissage vasculaire et à la noradrénaline, un syndrome de détresse respiratoire aiguë et une insuffisance rénale aiguë anurique. Ce tableau de défaillance multiviscérale conduira au 

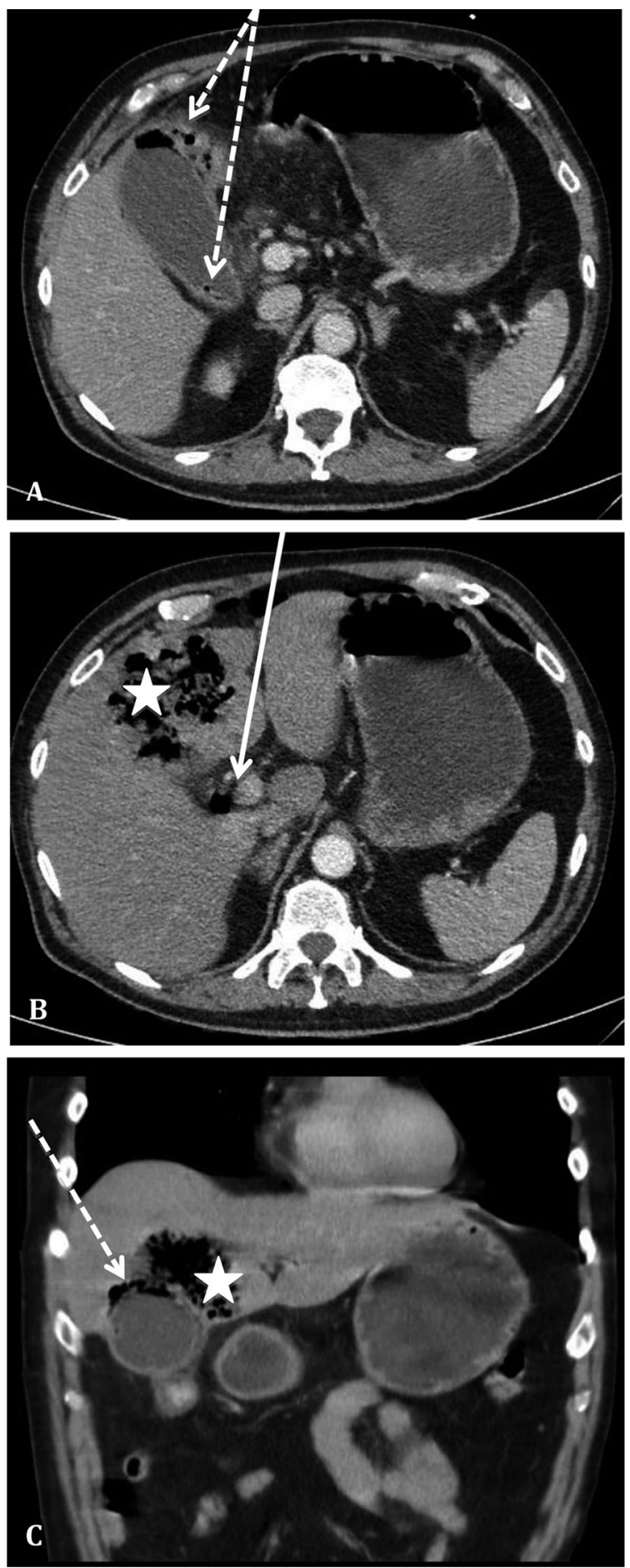

Fig. 1 TDM abdominopelvienne avec injection de produit de contraste. A. Coupe axiale : visualisation de bulles d'air intraet périvésiculaires (flèches pointillées). B. Coupe axiale : abcès hépatique périvésiculaire du segment IV (étoile), aérobilie (flèche pleine). C. Coupe coronale : abcès périhépatique du segment IV et emphysème sous-muqueux vésiculaire (flèche pointillée) décès du patient à 48 heures. Les cultures ont mis en évidence a posteriori une bactériémie à Clostridium perfringens.

\section{Discussion}

Au total, il s'agissait d'une péritonite biliaire compliquant une cholécystite emphysémateuse perforée à Clostridium perfringens avec abcès hépatique. Malgré un tableau clinique stable initialement, un choc septique réfractaire a aggravé la situation, entraînant une issue fatale. L'absence de signe digestif et de douleur abdominale a favorisé l'errance diagnostique et un retard de prise en charge. La décompensation d'un diabète de découverte récente au stade d'acidocétose a probablement favorisé cette complication septique. Plusieurs cas cliniques rapportent les observations de patients diabétiques victimes de cholécystites emphysémateuses ou d'abcès hépatiques, mais ces derniers présentaient souvent une douleur abdominale inaugurale et des signes d'irritation péritonéale [3,4]. De plus, il a récemment été montré que les pathologies cancéreuses, le diabète et les états immunodéprimés étaient des facteurs favorisants d'infections à Clostridium perfringens [5]. Les bactériémies à Clostridum perfringens sont des bactériémies très rares et de très mauvais pronostic. La réalisation d'hémocultures est nécessaire mais souvent peu contributive pour les bactéries anaérobies [1]. Quand le point de départ infectieux était retrouvé dans différents cas cliniques, il était principalement d'origine digestive et hépatobiliaire [5].

La prise en charge thérapeutique de cette complication consiste au contrôle de la source infectieuse par une antibiothérapie probabiliste à large spectre précoce et une prise en charge urgente au bloc opératoire [2].

Conflit d'intérêts : les auteurs déclarent ne pas avoir de liens d'intérêts.

\section{Références}

1. Shindo Y, Dobashi Y, Sakai T, et al (2015) Epidemiological and pathobiological profiles of Clostridium perfringens infections: review of consecutive series of 33 cases over a 13-year period. Int J Clin Exp Pathol 8:569-77

2. Cochrane J, Bland L, Noble M (2015) Intravascular hemolysis and septicemia due to Clostridium perfringens emphysematous cholecystitis and hepatic abscesses. Case Rep Med 2015 ;523402

3. Paasch C, Wilczek S, Strik MW (2017) Liver abscess and sepsis caused by Clostridium perfringens and Klebsiella oxytoca. Int J Surg Case Rep 41:180-3

4. Wu JM, Lee CY, Wu YM (2010) Emphysematous cholecystitis. Am J Surg 200:53-4

5. Lopez-Fabal MF, Sanz N, Ruiz-Bastian M, et al (2018) Clostridium perfringens bacteraemia, an analysis of 28 cases over 10 years in a university hospital of Madrid. Enferm Infecc Microbiol Clin 36:225-8 